\title{
Finding model parameters for the system waveform of a full-wave lidar: a pragmatic solution
}

Type: Conference contribution.

Status: Published October 10, 2018.

Version: Manuscript accepted for publication.

Copyright: Copyright 2018 Society of Photo-Optical Instrumentation Engineers (SPIE). One print or electronic copy may be made for personal use only. Systematic reproduction and distribution, duplication of any material in this publication for a fee or for commercial purposes, and modification of the contents of the publication are prohibited.

Bibliography: Roland Schwarz and Martin Pfennigbauer. "Finding model parameters for the system waveform of a full-wave lidar: a pragmatic solution." In: Remote Sensing of the Ocean, Sea Ice, Coastal Waters, and Large Water Regions 2018. Ed. by Charles R. Bostater, Stelios P. Mertikas, and Xavier Neyt. SPIE, Oct. 2018. DOI: 10.1117/12.2323965 



\title{
Finding model parameters for the system waveform of a full-wave lidar: a pragmatic solution
}

\author{
Roland Schwarz and Martin Pfennigbauer \\ Riegl Research Forschungs Gesellschaft mbH, 3580 Horn, Austria
}

\begin{abstract}
The system waveform (SWFM) of a pulsed LiDAR is obtained from the pulse shape received when pointing the sensor towards a flat, extended target with the surface normal equal to the laser beam direction. The SWFM is determined by the shape of the outgoing laser pulse and the transfer characteristics of the receiver. Knowing the SWFM is essential for performing highly accurate range measurements, for interpreting the LiDAR waveforms correctly, and to derive additional attributes for detected target returns. Often the actual SWFM is not known explicitly, and a Gaussian pulse shape is used in lieu thereof. However, the Gaussian pulse, despite its advantageous properties, does not properly address asymmetries and ringing effects typically present in real-life SWFMs. We present a model of the SWFM composed of harmonic and exponential terms which is able to account for these effects while at the same time being mathematically easy to handle. Unfortunately, the approximation of data by a sum of harmonics and exponentials belongs to the class of ill-posed problems. Nevertheless, we present a pragmatic solution to the problem and demonstrate the versatility of the resulting model.
\end{abstract}

Keywords: LiDAR, full-wave, waveform analysis, exponential model, system waveform, bathymetry

\section{INTRODUCTION}

The signal flow of a time-of-flight LiDAR system starts at a pulsed laser source. Fig. 1 shows a block diagram of a mono-static sensor where the transmitted pulse $P_{R}(t)$ and the received pulse $P_{E}(t)$ are collinear. At a range $R$, part of the pulse is reflected back as described by the differential back-scatter cross section $\operatorname{dBSCS} \sigma(R)$, resulting in

$$
P_{E}(t) \propto \int P_{T}\left(t-\frac{2 R}{v_{g}}\right) \sigma(R) d R
$$

with $v_{g}$ the group velocity in the medium at the frequency of the laser source. The received pulse is essentially proportional to the convolution of the transmitted pulse and the dBSCS, see e.g. Ref. 1.

A rotating mirror is used to deflect the beam to effect a scan of the remote target scene. Another system of mirrors is used to route the received beam to a photo detector.

At the detector the signal is converted from the optical domain to the electrical domain. The block diagram suggests that this conversion is a linear process that can be described by a factor $k$, a real number. Before quantizing and sampling the signal, the bandwidth must be limited by the filter $h(t)$ to avoid aliasing. Assuming linearity, the signal can be described by two convolutions where all proportionality factors have been subsumed into the single factor $k$

$$
s_{E}(t)=P_{T}(t) * \sigma(t) * k h(t) .
$$

By rearranging terms, we can identify the system waveform (SWFM) $p_{S}(t)$

$$
s_{E}(t)=\left(k h(t) * P_{T}(t)\right) * \sigma(t)=p_{S}(t) * \sigma(t)
$$

as the convolution of the outgoing pulse with the receivers impulse response. We note that if $\sigma(t)$ is approximately Dirac shaped, the signal $s_{E}(t)$ equals the SWFM.

\footnotetext{
Send correspondence to:

E-mail: office@riegl.com, Telephone: +43 29824211
} 


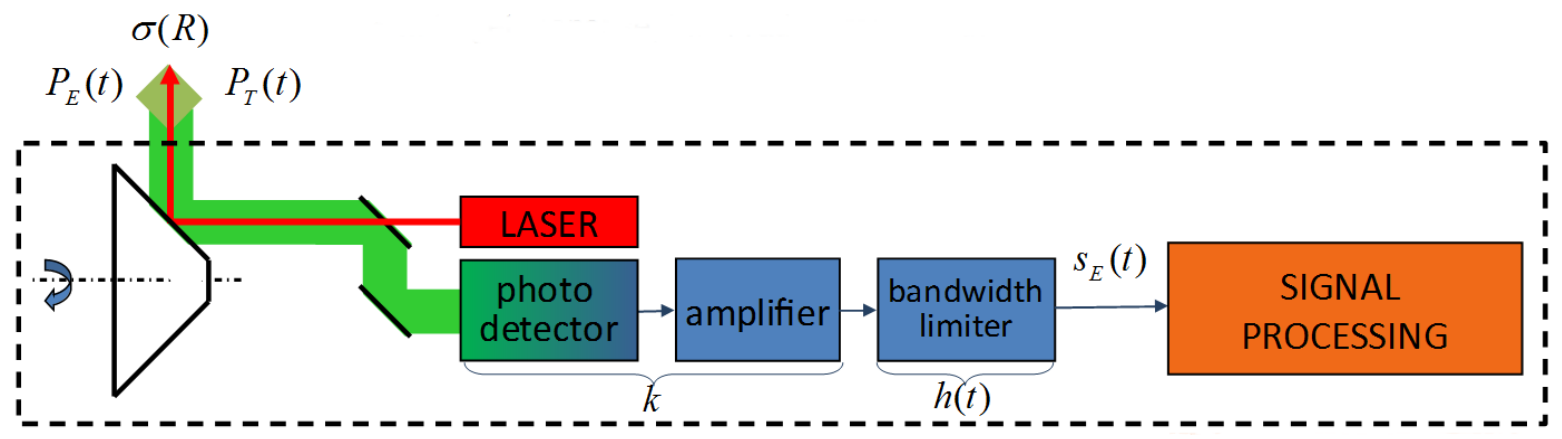

Figure 1. Signal flow of a pulsed LiDAR system. The transmitted laser pulse $P_{T}(t)$ is reflected by the range dependent differential backscatter cross-section $\sigma(R)$ and is received as a pulse $P_{E}(t)$. The pulse $P_{E}(t)$ is converted from the optical domain to the electrical domain, amplified by a factor $k$, and band-limited by a filter with impulse response $h(t)$. Finally, the electrical signal $s_{E}(t)$ is converted to the digital domain for further processing.

Deconvolution is the process of retrieving the dBSCS from a recording of the received waveform. For the application of deconvolution either explicit knowledge of the SWFM is required ${ }^{2}$ or the SWFM must be assumed ${ }^{3}$ implicitly, usually as Gaussian shaped. It has been proposed to use a B-spline model ${ }^{4}$ for the SWFM because of its mathematical properties. We propose a model of the SWFM composed of a sum of complex exponentials because of its ability to express the convolution with an exponential segment in explicit form. ${ }^{5}$

The mathematical problem of approximating a measured function with a sum of exponentials is well known to belong into the range of numerically difficult ${ }^{6}$ problems. The problem can be dated back to Prony, who published a method for the solution in 1795. Osborne and Smyth presented an algorithm for exponential function fitting ${ }^{7}$ in 1995. As it turned out the algorithms performed poorly on our data. The question as for the exact reasons remains unanswered to us for now. It might be that the number of data samples was too large (about 400) for successful application or that the combination of strong damping coefficients and sinusoids at the same time constituted a problem. However, this is why we aimed for a pragmatic approach based on classical non-linear optimization techniques. The main contribution of this presentation is the construction of good initial values for the optimization, so that the iteration will converge with high probability.

\section{METHOD}

\subsection{Linear part of the system waveform}

A Dirac shaped $\sigma(t)$ is realized when targeting the laser towards a flat, extended target in perpendicular direction. In theory, following from linearity, a single pulse of sufficient energy should then be enough to characterize the whole system. However, for any realizable system linearity holds only to a certain degree, and as such for higher pulse amplitudes there is a deviation from linearity. In order to operate a sensor even in this non-linear range, the SWFMs are recorded also for high power signals ${ }^{8}$ as part of the calibration procedure. The linear part of the SWFM can be derived from these waveforms.

Fig. 2, left, shows the SWFM $p_{S}(A, t)$ for excitation pulses $p_{T}(t)$ of varying amplitudes $A$. A linear subset from these pulses can be selected by noting that a necessary criterion for linearity is that for any two amplitudes $A_{i}, A_{j}$ the waveforms must satisfy a proportionality of

$$
p_{S}\left(A_{j}, t\right)=k_{i j} p_{S}\left(A_{i}, t\right)+d_{i j}
$$

for fixed constants $k_{i j}$ and $d_{i j}$. This problem can be readily solved by calculating the Pearson's correlation coefficient for any combination of $i$ and $j$ and selecting the subset where the coefficient surpasses a given threshold for all indices. Fig. 2, right, shows a set of traces selected by this procedure. The next step is to generate the linear prototype SWFM from these traces by averaging. Before averaging each trace, $p_{S}\left(A_{j}, t\right)$ is normalized as

$$
p_{N}\left(A_{j}, t\right)=\frac{p_{S}\left(A_{j}, t\right)-\overline{p_{S}\left(A_{j}, t\right)}}{\left|p_{S}\left(A_{j}, t\right)-\overline{p_{S}\left(A_{j}, t\right)}\right|},
$$



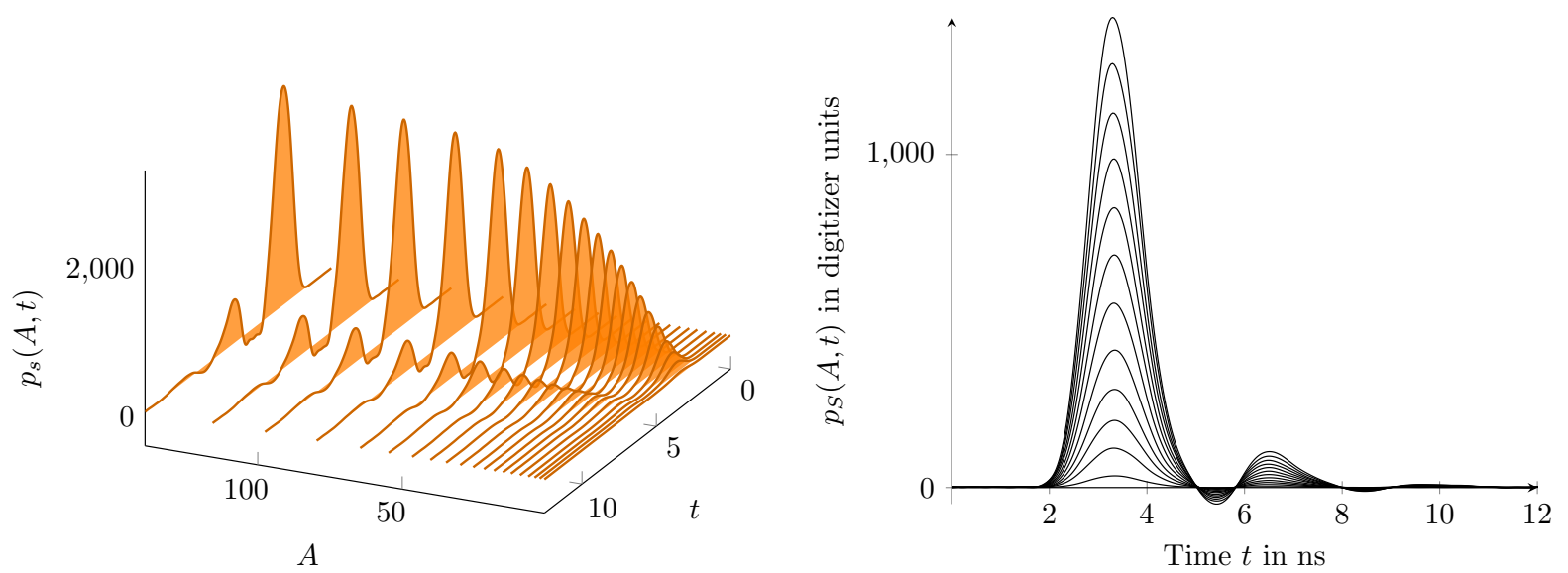

Figure 2. Nonlinear and linear recordings of $s_{E}(t)$ when pointing the laser towards a flat, extended target. Left: Recorded traces of the received signal $s_{E}(t)$ for different relative power levels $A$. Right: For levels of low power the traces exhibit a linear similarity, which can be found by cross correlation.

where the mean and magnitude operations are defined by $\overline{x(t)}=\frac{1}{T} \sum_{t=0}^{T} x(t)$ and $|x(t)|=\sqrt{\sum_{t=0}^{T} x^{2}(t)}$. The chosen kind of normalization makes the result immune to errors from offset. Finally, assuming the number of traces is $J$, the SWFM

$$
p_{S}(t)=k\left(\frac{1}{J} \sum_{j=1}^{J} p_{N}\left(A_{j}, t\right)\right)+d
$$

is the mean of the normalized traces with $k$ and $d$ chosen so that the start of the trace equals zero and the maximum equals one, as can be seen in the left image of Fig. 3.
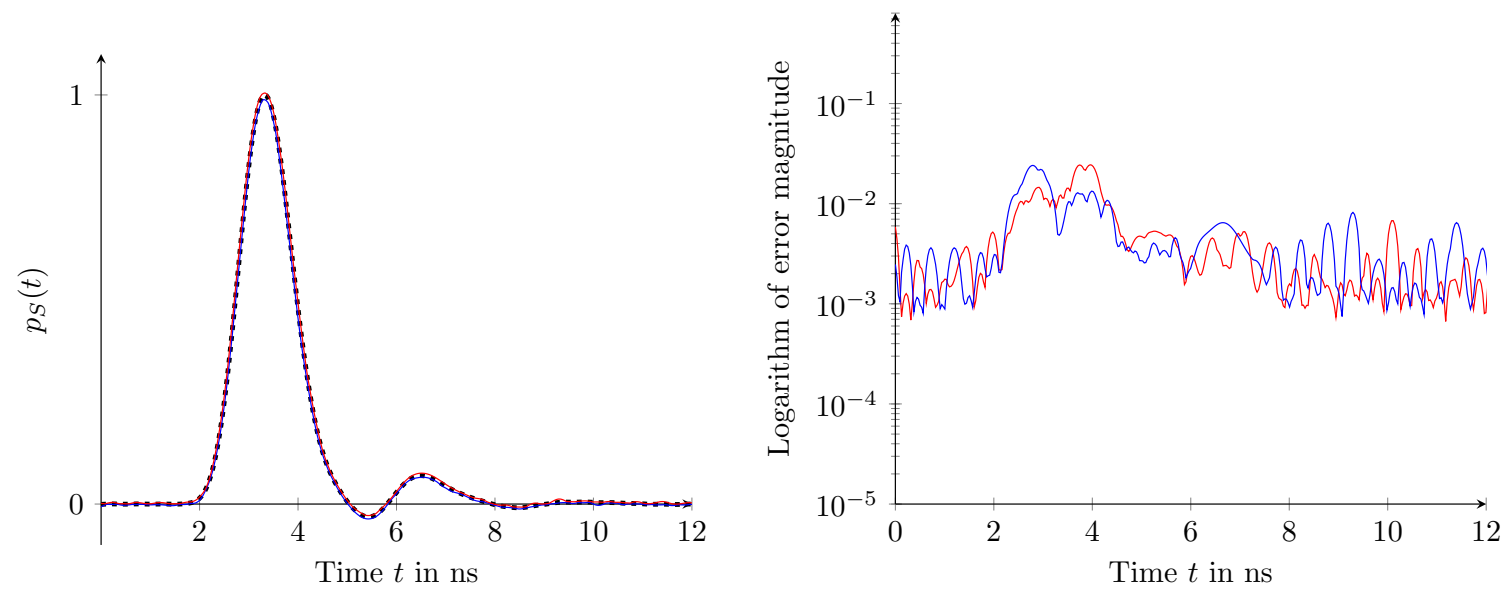

Figure 3. Linear SWFM. Left: The SWFM is the mean, marked by dots, of all normalized traces belonging to the linear range. The maximum and minimum of all traces are overlaid in red and blue respectively. Right: Upper maximum error (red) and lower maximum error (blue) of the normalized traces from the mean trace.

To illustrate the effectiveness of the procedure, the upper and lower bounds of all traces, scaled by the same $k$ and $d$, have been super-imposed on the SWFM. Since the differences are too small to be viewable in the same graph as $p_{S}(t)$, the right image of Fig. 3 shows a logarithmic plot of the differences in two colors, with upper bound minus $p_{S}(t)$ in red and lower bound minus $p_{S}(t)$ in blue. 


\subsection{Sum of causal complex exponentials}

A common approach to modeling the SWFM is by means of a function of Gaussian shape. This has a lot of mathematical, theoretical, and practical advantages, but it also has its shortcomings. The Gaussian shape is inherently symmetric with respect to its mean value and it is never exactly zero. The latter, although usually not a problem in practice, has the theoretical implication that the Gaussian shaped function does not describe a causal system because it is not zero for $t<0$. A class of functions which are causal and can be expected to be suitable for modeling of the SWFM can be defined as the real part of a sum of $I$ truncated exponentials

$$
p(t)=u(t) \Re\left(\sum_{i=0}^{I-1} a_{i} e^{\alpha_{i} t}\right)
$$

with complex parameters $a_{i}$ and $\alpha_{i}$. The unit step function $u(t)$ is zero for $t<0$ and one everywhere else. By noting that the real part of a complex number is $\Re(x)=\frac{1}{2}\left(x+x^{*}\right)$ and the settings $\alpha=\gamma+\mathrm{i} \omega$ and $a=A e^{\mathrm{i} \varphi}$, Eq. 7 can alternatively be written as

$$
p(t)=u(t) \sum_{i=0}^{I-1} A_{i} e^{\gamma_{i} t} \cos \left(\omega_{i} t+\varphi_{i}\right) .
$$

In order to arrive at $p(t)=0$ for $t \rightarrow+\infty$ all $\gamma_{i}$ or equivalently all $\Re\left(\alpha_{i}\right)$ must be negative.

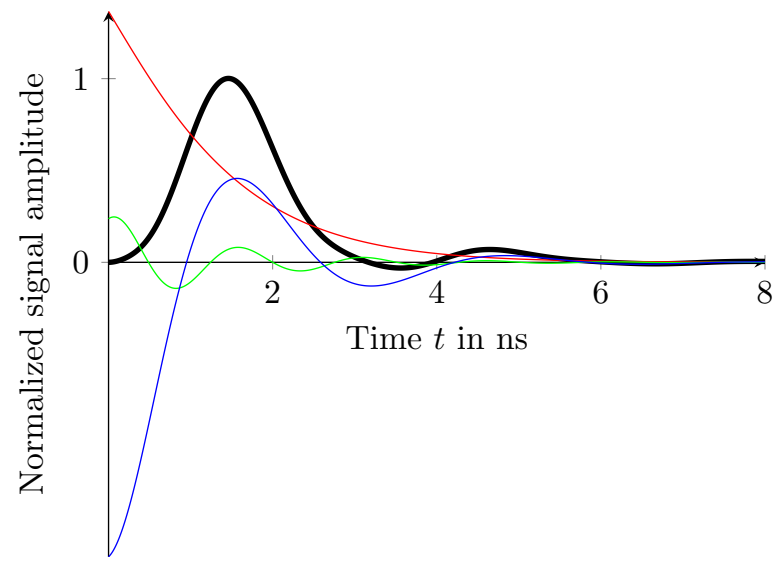

Figure 4. An exponential sum consisting of three terms. The components in red, green, and blue add up to the pulse shape of $p(t)$ in black. At the beginning the sum is forced to zero, and for $t \rightarrow+\infty$ all components are exponentially damped. For time instances $t<0$ the sum is zero by definition.

Fig. 4 shows the components, in color, and the sum, in black, of a three-term causal exponential sum. At time $t=0$ the parameters must be chosen such that the terms add up to zero. The terms are not evaluated for $t<0$ since in this case the function is zero by definition.

\subsection{Finding the parameters of the exponentials to match the SWFM}

The function model described by Eq. 7 is matched to the data given by Eq. 6 with a non-linear parameter fit based on the method of Levenberg-Marquardt (LM). The method is not new and has been described in the literature and it is well known that a very good estimate of the initial parameters is required for convergence (see e.g. Ref. 6). The adjective pragmatic in the title of this paper is attributed to the manner in which such good initial values of the parameters can be found.

The very first step is to shift the time axis such that the start of the pulse will occur shorty after time instant zero, see Fig. 4. The precise location of the beginning will consequently be adjusted by making the start instant a parameter of the fitting procedure. 
Although we will later see that the simplest model, which is able to describe a causal pulse, consists of only a single term we start with the following 3 parameter, two term, model

$$
p_{1}(t)=a\left(e^{\alpha t}-e^{\beta t}\right)
$$

with parameters $a, \alpha$, and $\beta$. We note that $p_{1}(0)=0$ for $t=0$. By means of the Fourier transform one can further show that

$$
M_{n}=\int_{0}^{\infty} t^{n} p_{1}(t) d t=(-1)^{n+1} n ! a\left(\frac{1}{\alpha^{n+1}}-\frac{1}{\beta^{n+1}}\right) .
$$

It is worth noting that the integral to the left of Eq. 10 is similar to the definition of statistical or mechanical moments. However, because $p_{1}(t)$ need not be a strictly positive function, the sign of $M_{n}$ also may be negative. Being aware of the apparent difference to the mechanical moments nevertheless we define

$$
\begin{aligned}
& M_{0}=A \\
& M_{1}=\mu A \\
& M_{2}=A\left(\sigma^{2}+\mu^{2}\right)
\end{aligned}
$$

with $A$ corresponding to the area, $\mu$ the center of gravity and $\sigma$ the deviation. Now the following relations between the parameters an the moments can be deduced:

$$
\begin{aligned}
a & =\frac{A}{\sqrt{2 \sigma^{2}-\mu^{2}}} \\
\alpha & =\frac{1}{\left(\mu^{2}-\sigma^{2}\right)}\left(-\mu+\sqrt{2 \sigma^{2}-\mu^{2}}\right) \\
\beta & =\frac{1}{\left(\mu^{2}-\sigma^{2}\right)}\left(-\mu-\sqrt{2 \sigma^{2}-\mu^{2}}\right) .
\end{aligned}
$$

If $2 \sigma^{2}<\mu^{2}$, the square root of Eq. 14 is purely imaginary, and so is $a$. This means that the two terms in braces of Eq. 9 are conjugate complex, which is why the sum can be represented as a single term of Eq. 7 in that case.

Equating $A, \mu$, and $\sigma$ with the moments of the sampled signal

$$
\begin{aligned}
A & =\sum_{t=0}^{T} p_{s}(t) \\
\mu & =\frac{1}{A} \sum_{t=0}^{T} t p_{s}(t) \\
\sigma^{2} & =\frac{1}{A} \sum_{t=0}^{T}(t-\mu)^{2} p_{s}(t)
\end{aligned}
$$

we get a first order approximation for the parameters. In Fig. 5, left image, it can be seen that the approximation (red line) to the SWFM (dotted) clearly is not very good yet.

Proceeding by taking the difference of $p_{S}(t)-p_{1}(t)$ we get a pulse like the one that can be seen in the right image of Fig. 5, red line. We recognize that, because $p_{S}(t)$ and $p_{1}(t)$ have effectively the same area, the difference must be oscillatory. Thus it is reasonable to model the difference as a harmonic modulated by $p_{1}(t)$

$$
p_{S}(t)-p_{1}(t)=p_{1}(t) B \cos (\omega t+\varphi)
$$

The frequency $\omega$ can be deduced from the zero crossings, the amplitude $B$ by matching the maximum and the phase $\varphi$ by shifting the cosine so that the maxima occur at the same time instant. The blue line in the right image of Fig. 5 shows the result of such a choice of parameters. The second order initial value is then given as

$$
p_{2}(t)=p_{1}(t)(1+B \cos (\omega t+\varphi)) .
$$



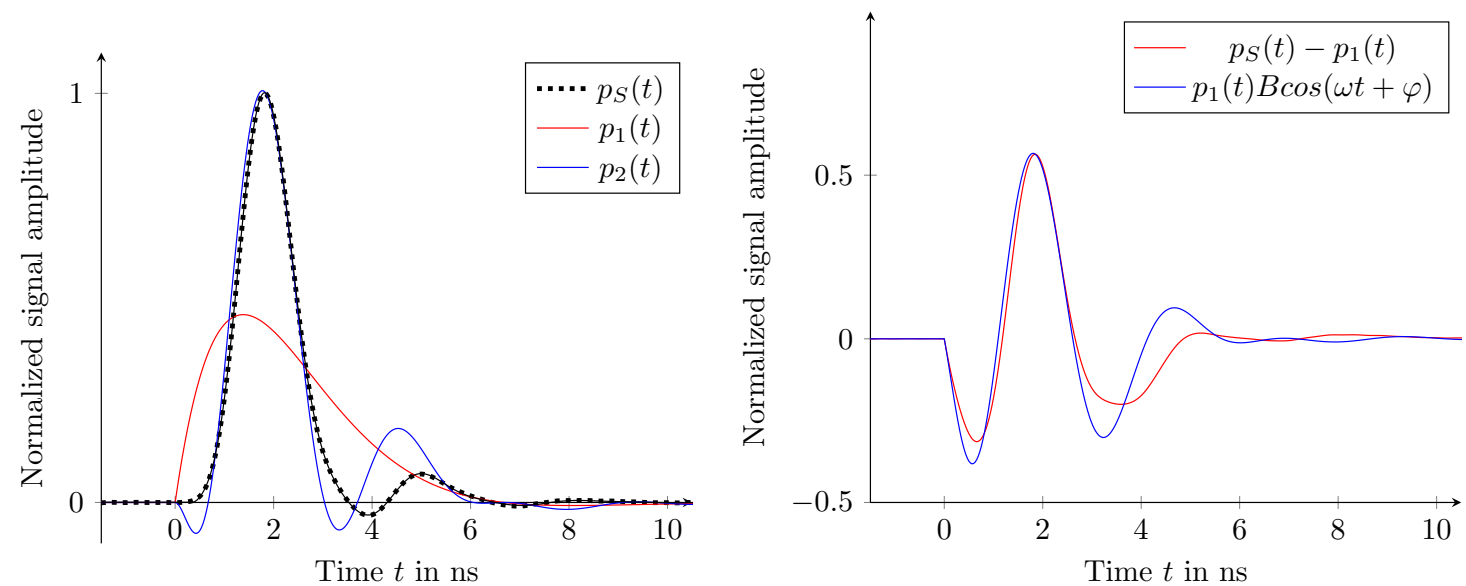

Figure 5. Finding the parameters of the sum of causal exponentials. The dotted line in the left image is the SWFM defined by the sample values $p_{S}(t)$. The red and blue lines are the first $p_{1}(t)$ and second order $p_{2}(t)$ approximations for the initial values of non-linear parameter fit. The image on the right shows the difference of the sample values $p_{S}(t)$ and the first order approximation (red line) and a model of the difference (blue line) according to Eq. 20.

The right hand side of Eq. 21 can be put in the form of Eq. 7, i.e., an exponential sum. The blue line of the left image in Fig. 5 indicates that $p_{2}(t)$ is now a much better initial value.

The remainder of the algorithm is a standard parameter fit with a few constraints to be imposed

$$
\begin{aligned}
& \sum_{i=0}^{I-1} a_{i}=0 \\
& \Re\left(\alpha_{i}\right)<0 .
\end{aligned}
$$

If after convergence the fit is not accurate enough it is possible to increase the order of the model by adding another term. We have seen that zero initializing these additional parameters usually will give satisfying results.

\section{RESULTS AND DISCUSSION}

Owing to the pragmatic approach, the authors did not attempt to give a mathematical proof for the convergence of the method or the uniqueness of the solution. Instead the method was tested for a range of SWFMs from different types of RIEGL instruments with good results. Tab. 1 lists 20 samples of four instrument types. The algorithm succeeded in all cases. The remaining columns are a $2 \sigma$ value, reminiscent of the pulse width as known from the Gaussian pulse shape, the number of terms $N$ chosen by the algorithm, the root-mean-square-error (RMSE) between the model and the data, and the maximum error between the model and data. As can be seen in sample 17 and 18 of Tab. 1 , since $\sigma^{2}$ need not be a positive number if the pulse is not strictly positive, the $2 \sigma$ value may turn complex. Despite this fact, which might be perplexing at first, the algorithm is still able to converge to a good approximation of the SWFM. Considering that the maximum of the pulse has been scaled to one, the error figures show that it is possible to approximate the SWFM by a few exponential terms resulting in errors consistently less than $10^{-2}$. This is comparable to the deviations between the mean sample SWFM and the other traces, as can bee seen in Fig. 3.

\section{CONCLUSIONS AND OUTLOOK}

The findings presented here indicate that it is possible to approximate the SWFM of a laser sensor by a few exponential terms. Similar to the approximation with a Gaussian pulse shape, the exponential sum is a sample rate free, i.e. a continuous, description of the SWFM. For a good model of the SWFM it is desirable that the operation of convolution can be carried out explicitly. It is well known that the convolution of two Gaussian 
Table 1. Pulse model estimation results for 20 RIEGL instruments SWFMs of various types. RMSE is the root-meansquare between data and model, $\varepsilon_{\max }$ is the magnitude of the largest error, $\mathrm{N}$ is the number of exponential terms, and $2 \sigma$ is a measure of pulse width. Note: $2 \sigma$ may become complex, see discussion in the text.

\begin{tabular}{|c|l|l|l|l|l|}
\hline Sample & Type & \multicolumn{1}{|c|}{$\boldsymbol{\sigma}$} & $\mathbf{N}$ & $\mathbf{R M S E}$ & $\boldsymbol{\varepsilon}_{\mathbf{m a x}}$ \\
\hline 1 & VQ-880-G-IR & $2.81 \mathrm{~ns}$ & 4 & 0.002 & 0.01 \\
\hline 2 & VQ-880-G-IR & $3.03 \mathrm{~ns}$ & 4 & 0.002 & 0.01 \\
\hline 3 & VQ-880-G-IR & $3.02 \mathrm{~ns}$ & 4 & 0.002 & 0.008 \\
\hline 4 & VQ-880-G-IR & $3.04 \mathrm{~ns}$ & 4 & 0.002 & 0.01 \\
\hline 5 & VQ-880-G-IR & $3.00 \mathrm{~ns}$ & 4 & 0.002 & 0.008 \\
\hline 6 & VQ-880-G-IR & $3.01 \mathrm{~ns}$ & 4 & 0.002 & 0.009 \\
\hline 7 & VQ-880-G-IR & $2.94 \mathrm{~ns}$ & 4 & 0.002 & 0.009 \\
\hline 8 & VQ-880-G & $3.48 \mathrm{~ns}$ & 4 & 0.002 & 0.004 \\
\hline 9 & VQ-880-G & $3.49 \mathrm{~ns}$ & 4 & 0.002 & 0.004 \\
\hline 10 & VQ-880-G & $3.48 \mathrm{~ns}$ & 4 & 0.002 & 0.005 \\
\hline 11 & BDF-1 & $1.76 \mathrm{~ns}$ & 3 & 0.001 & 0.004 \\
\hline 12 & BDF-1 & $1.79 \mathrm{~ns}$ & 3 & 0.001 & 0.004 \\
\hline 13 & BDF-1 & $1.76 \mathrm{~ns}$ & 3 & 0.001 & 0.004 \\
\hline 14 & BDF-1 & $1.72 \mathrm{~ns}$ & 3 & 0.001 & 0.005 \\
\hline 15 & BDF-1 & $1.71 \mathrm{~ns}$ & 3 & 0.001 & 0.005 \\
\hline 16 & BDF-1 & $2.55 \mathrm{~ns}$ & 4 & 0.0005 & 0.002 \\
\hline 17 & VQ-1560i & $5.50 \mathrm{ins}$ & 4 & 0.001 & 0.005 \\
\hline 18 & VQ-1560i & $7.22 \mathrm{ins}$ & 4 & 0.003 & 0.008 \\
\hline 19 & VQ-1560i & $4.88 \mathrm{~ns}$ & 3 & 0.001 & 0.004 \\
\hline 20 & VQ-1560i & $4.94 \mathrm{~ns}$ & 3 & 0.001 & 0.003 \\
\hline
\end{tabular}

functions yields a Gaussian shaped result. A corresponding statement holds for the convolution of two exponential sums. Other function types are possible: the convolution of exponential sums with Dirac-, exponential-, and boxcar-shaped functions has already been used successfully by the authors to decompose bathymetric signals. ${ }^{5}$

The Beer-Lambert law, essentially stating exponential attenuation behavior of light in media, lets us expect that the description of the SWFM by an exponential sum will enable application of our method in areas beyond bathymetry as well.

\section{REFERENCES}

[1] Wagner, W., Ullrich, A., Ducic, V., Melzer, T., and Studnicka, N., "Gaussian decomposition and calibration of a novel small-footprint full-waveform digitising airborne laser scanner," ISPRS Journal of Photogrammetry and Remote Sensing 60, 100-112 (apr 2006). bibtex: wagner_gaussian_2006.

[2] Cawse-Nicholson, K., van Aardt, J., Hagstrom, S., Romanczyk, P., Schaaf, C., Strahler, A., Li, Z., and Krause, K., "Improving waveform lidar processing toward robust deconvolution of signals for improved structural assessments," in [Laser Radar Technology and Applications XIX and Atmospheric Propagation XI], Turner, M. D., Kamerman, G. W., Wasiczko Thomas, L. M., and Spillar, E. J., eds., 90800I, SPIE (jun 2014).

[3] Wu, J., van Aardt, J. A. N., and Asner, G. P., "A comparison of signal deconvolution algorithms based on small-footprint LiDAR waveform simulation," IEEE Transactions on Geoscience and Remote Sensing 49, 2402-2414 (jun 2011). 
[4] Roncat, A., Bergauer, G., and Pfeifer, N., "B-spline deconvolution for differential target cross-section determination in full-waveform laser scanning data," 66(4), 418-428. bibtex: roncat_b-spline_2011.

[5] Schwarz, R., Pfeifer, N., Pfennigbauer, M., and Ullrich, A., "Exponential decomposition with implicit deconvolution of lidar backscatter from the water column," PFG - Journal of Photogrammetry, Remote Sensing and Geoinformation Science 85, 159-167 (jul 2017).

[6] Kannan, N. and Kundu, D., "Estimating parameters in the damped exponential model," Signal Processing 81, 2343-2351 (nov 2001).

[7] Osborne, M. and Smyth, G., "A modified prony algorithm for exponential function fitting," 16(1), $119-138$. bibtex: osborne_modified_1995.

[8] Ullrich, A. and Pfennigbauer, M., "Echo digitization and waveform analysis in airborne and terrestrial laser scanning," in [Photogramm. Week 2011], Photogrammetric Week, 217-228 (2011). 